\title{
Effect of Food Intake on Hemodynamic Parameters during the Tilt-Table Test in Patients with Postural Orthostatic Tachycardia Syndrome
}

\author{
Mario Habek ${ }^{a, b}$ \\ Berislav Ruška ${ }^{a}$ \\ Luka Crnošija ${ }^{b}$ \\ Ivan Adamec ${ }^{\mathrm{b}}$ \\ Anamari Junakovićb \\ Magdalena Krbot Skorićb \\ aDepartment of Neurology, \\ School of Medicine, \\ University of Zagreb, \\ Zagreb, Croatia \\ bDepartment of Neurology, \\ University Hospital Center Zagreb, \\ Referral Center for Autonomic Nervous \\ System Disorders, Zagreb, Croatia
}

\begin{abstract}
Background and Purpose The aim of this study was to determine the effect of food intake on the heart rate (HR) in postural orthostatic tachycardia syndrome (POTS).

Methods The following five-phase protocol was applied to 41 subjects who had a sustained HR increment of $\geq 30$ beats/min within $10 \mathrm{~min}$ of standing in an initial tilt-table test: 1) 10min supine phase, 2) 10 -min $70^{\circ}$-tilted phase, 3) ingestion of $400 \mathrm{~mL}$ of Nutridrink Multi Fibre $^{\circledR}$, 4) 45 -min supine phase, and 5) 10-min $70^{\circ}$-tilted phase. Subjects were divided into four groups: A) difference in HR for standing vs. supine ( $\triangle \mathrm{HR}$ ) before the meal of $\geq 30$ beats $/ \mathrm{min}$ $(n=13)$, B) $\Delta$ HR $<30$ beats $/$ min before the meal but $\geq 30$ beats $/ \mathrm{min}$ after the meal $(n=12)$, and C) $\Delta \mathrm{HR}<30$ beats/min both before and after the meal ( $n=16)$. Group D consisted of 10 healthy subjects.

Results Before the meal, $\triangle \mathrm{HR}$ was significantly higher in group $\mathrm{A}$ than in all of the other groups, and in group $\mathrm{B}$ than in group $\mathrm{D}(p<0.001)$. After the meal, $\Delta \mathrm{HR}$ was significantly higher in groups $\mathrm{A}$ and $\mathrm{B}(p<0.001$ and $p<0.0001$, respectively). When we pooled patients (according to their symptoms) from group A and B into a POTS group and from group C and D into a non-POTS group, an increase in HR of 25 beats/min before the meal had a sensitivity of $92.0 \%$ and a specificity of $80.8 \%$. After the meal, an increase in HR of 30 beats $/ \mathrm{min}$ had a sensitivity of $96.0 \%$ and a specificity of $96.2 \%$.

Conclusions Food intake can significantly alter the results of the tilt-table test and so should be taken into account when diagnosing POTS.
\end{abstract}

Key Words postural orthostatic tachycardia syndrome, food intake, tilt-table test.
Received June 27, 2018

Revised November 26, 2018

Accepted November 28, 2018

\section{Correspondence}

Mario Habek, MD, PhD

Department of Neurology,

University Hospital Center Zagreb,

Referral Center for Autonomic Nervous

System Disorders, Kišpatićeva 12,

HR-10000 Zagreb, Croatia

Tel +38512388033

Fax +38512388033

E-mail mhabek@mef.hr

\section{INTRODUCTION}

Postural orthostatic tachycardia syndrome (POTS) is a form of orthostatic intolerance characterized by a sustained excessive increase in the heart rate (HR) after adopting an upright posture. The HR increase in POTS is accompanied by characteristic symptoms such as lightheadedness, weakness, and nausea, which are relieved by adopting a recumbent position. ${ }^{1}$ This abnormal response to orthostasis could be pathophysiologically explained by insufficient compensatory vascular constriction, excessive central sympathetic tone, hypovolemia with a defect in the renin-angiotensin-aldosterone system, deconditioning, and autoimmune causes, but the exact underlying mechanism remains unknown. ${ }^{1,2}$ POTS is more common among females and either can appear in isolation or can overlap and be secondary to other conditions such as chronic fatigue syndrome, multiple sclerosis, inappropriate sinus tachycardia, and Ehlers-Danlos syndrome. ${ }^{1,3,4}$ The exact prevalence of POTS is unknown, mostly because many patients who suffer from this condition have unspecific symptoms,

(a) This is an Open Access article distributed under the terms of the Creative Commons Attribution Non-Commercial License (https://creativecommons.org/licenses/by-nc/4.0) which permits unrestricted non-commercial use, distribution, and reproduction in any medium, provided the original work is properly cited. 
resulting in them being underdiagnosed or misdiagnosed and hence treated for some other condition instead. ${ }^{5}$ A definitive diagnosis of POTS requires fulfillment of the following criteria reported in 2011 by Freeman et al., ${ }^{6}$ a sustained HR increment of $\geq 30$ beats/min within $10 \mathrm{~min}$ of standing or during head-up tilting in the absence of orthostatic hypotension and accompanied by characteristic symptoms that are relieved by recumbency. For individuals aged 12-19 years, the required minimum increment in HR is 40 beats $/ \mathrm{min}^{6}{ }^{6}$ The tilttable test is a common diagnostic tool for diagnosing POTS because it enables the positions of the patient to be readily altered between standing and supine while measuring the blood pressure (BP) and HR changes, which are major criteria for POTS.

Meal intake significantly influences the autonomic nervous system, and it is estimated that maintaining the postprandial BP requires a $200 \%$ increase in sympathetic nervous activity. ${ }^{7,8}$ This effect of the sympathetic nervous response seems to be even greater in young adults, which is the population mostly affected by POTS. ${ }^{9}$ Since several neurological illnesses can influence the postprandial response, it is no wonder that gastroenterologic symptoms are common in the POTS, and postprandial worsening of orthostatic symptoms often occurs. ${ }^{10,11}$ One study has shown how food ingestion alters the HR and BP upon head-up tilting in POTS patients. ${ }^{11}$ However, current POTS criteria do not define whether food intake should be taken into account while interpreting the results of tilt-table tests. Based on the hypothesis that food intake prior to tilt-ta- ble testing can influence the criteria used to diagnose POTS, the aim of this study was to determine the effect of food intake on the current criteria applied for the diagnosis of POTS.

\section{METHODS}

This was a prospective study that was conducted from April 2016 to November 2017 in the Referral Center for Autonomic Nervous System Disorders at University Hospital Center Zagreb.

Eligible participants were consecutive patients referred to the center for tilt-table testing and who had a sustained HR increment of $\geq 30$ beats/min within $10 \mathrm{~min}$ of standing or during head-up tilting in the absence of orthostatic hypotension with or without characteristic symptoms for POTS in the baseline examination. ${ }^{6}$ Exclusion criteria were the presence of any underlying condition that might influence the results in the tilt-table test such as hypertension, diabetes or polyneuropathy. Furthermore, age- and sex-matched healthy controls who had no previous history of events that could be associated with any kind of orthostatic intolerance were included. All participants signed informed-consent forms approved by the Ethical Committee of University Hospital Center Zagreb (approval no. 02/21 AG).

Forty-one participants met the inclusion criteria (29 females; age $29.74 \pm 10.61$ years, mean $\pm S D)$, and the control group consisted of 10 healthy individuals ( 6 females, age $24.25 \pm$ 2.25 years). The study flow chart is presented in Fig. 1. After

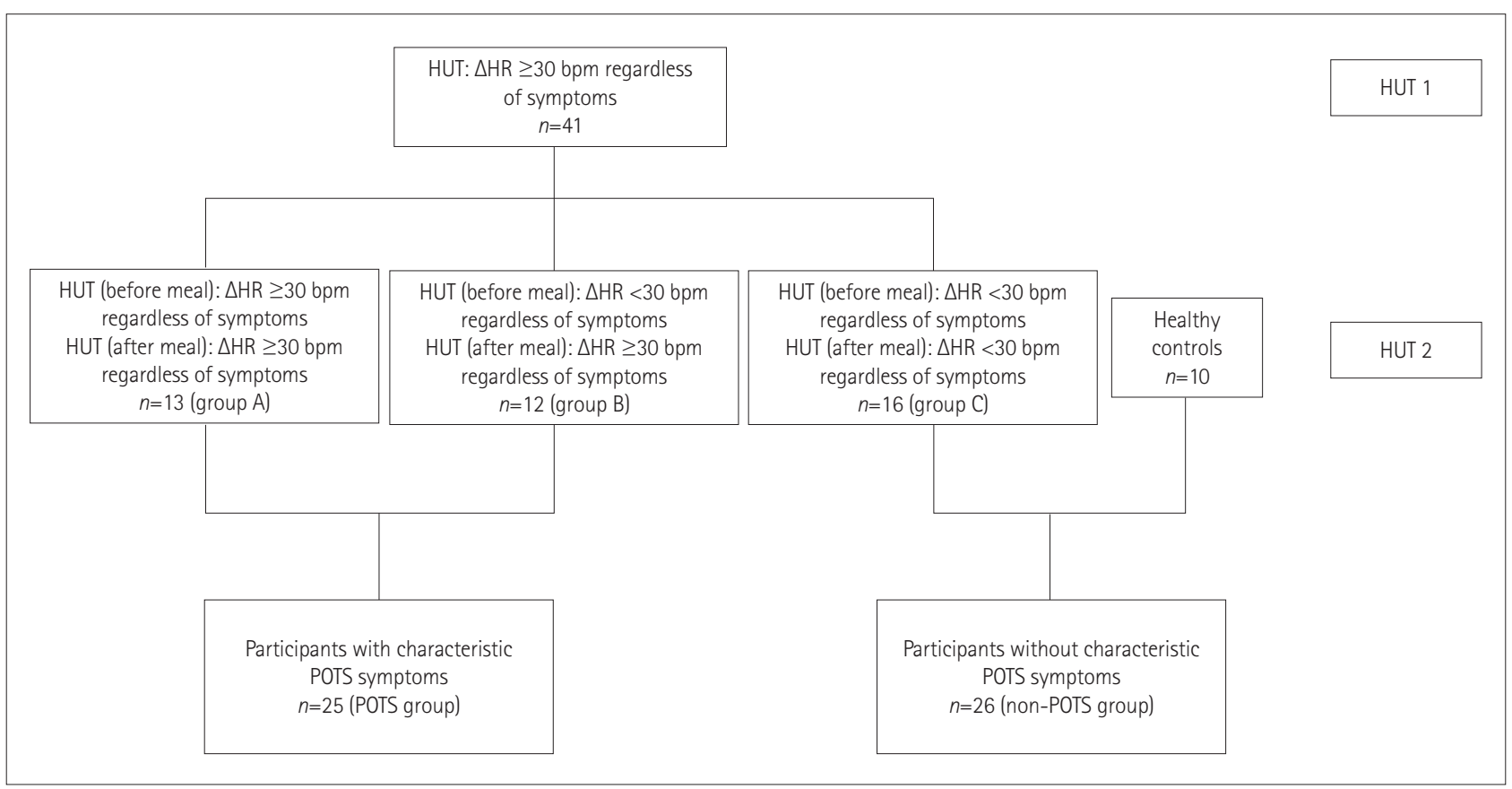

Fig. 1. Study flow chart. HR: heart rate, $\triangle H R$ : difference in heart rate for tilted vs. supine, HUT: head-up tilt-table test, POTS: postural orthostatic tachycardia syndrome. 
fulfilling the inclusion criteria, participants were asked to come to the laboratory within 28 days, when they underwent the second tilt-table test comprising the following five phases: 1) 10-min supine phase, 2) 10 -min $70^{\circ}$-tilted phase, 3 ) meal ingestion (5-7 min), 4) 45-min supine phase, 5) $10-\min 70^{\circ}$ -tilted phase.

The beat-to-beat $\mathrm{HR}$ and BP were continuously monitored throughout the test using a Task Force Monitor (CNSystems Medizintechnik, Graz, Austria). The meal consumed by the participants comprised $400 \mathrm{~mL}$ of the liquid meal replacement Nutridrink Multi Fibre ${ }^{\circledR}$ at room temperature. One $200-\mathrm{mL}$ pack has an energy content of $300 \mathrm{kcal}(100 \mathrm{~mL}$ contains $5.8 \mathrm{~g}$ of fat, $6 \mathrm{~g}$ of protein, and $18 \mathrm{~g}$ of carbohydrate). All of the participants had fasted for at least 3 hours prior to the testing. None of the participants received any form of treatment between testing that could affect their POTS symptoms.

In order to investigate the effect of food intake on hemodynamic parameters during the tilt-table test, we divided subjects into four groups: A) difference in HR for standing vs. supine $(\Delta \mathrm{HR})$ before the meal of $\geq 30$ beats $/ \mathrm{min}(n=13)$, B) $\Delta \mathrm{HR}<30$ beats $/ \mathrm{min}$ before the meal but $\geq 30$ beats $/ \mathrm{min}$ after the meal $(n=12)$, C) $\Delta \mathrm{HR}<30$ beats/min both before and after the meal $(n=16)$, and D) healthy subjects $(n=10)$ (Fig. 2).

In order to test the hypothesis, we pooled patients from group A and B into a POTS group and patients from group $\mathrm{C}$ and $\mathrm{D}$ into a non-POTS group according to their symptoms. $^{12}$

Statistical analysis was conducted using the SPSS software (version 20; IBM Corp., Armonk, NY, USA). The Kolmogorov-Smirnov test was used to check whether the data conformed to a normal distribution. Differences in the distribution of qualitative variables were determined using the $\chi^{2}$ test, while differences in quantitative variables were determined using the ANOVA with the Bonferroni post-hoc multiple-comparison correction, and using the paired $t$-test. For the paired $t$-test, according to Bonferroni multiple-comparison correction, a $p$ value of $<0.001$ was considered statistically significant. For other comparisons, a $p$ value $<0.05$ was considered statistically significant. Receiver operating characteristics (ROC) curves were used to interpret the sensitivity and specificity of different cutoff values in differentiating between POTS and non-POTS patients.

\section{RESULTS}

Descriptive data and the distributions of HR and BP parameters before and after the meal are presented in Table 1. All of the participants in groups A and B had a 3-month history of symptoms characteristic of POTS. Participants from group

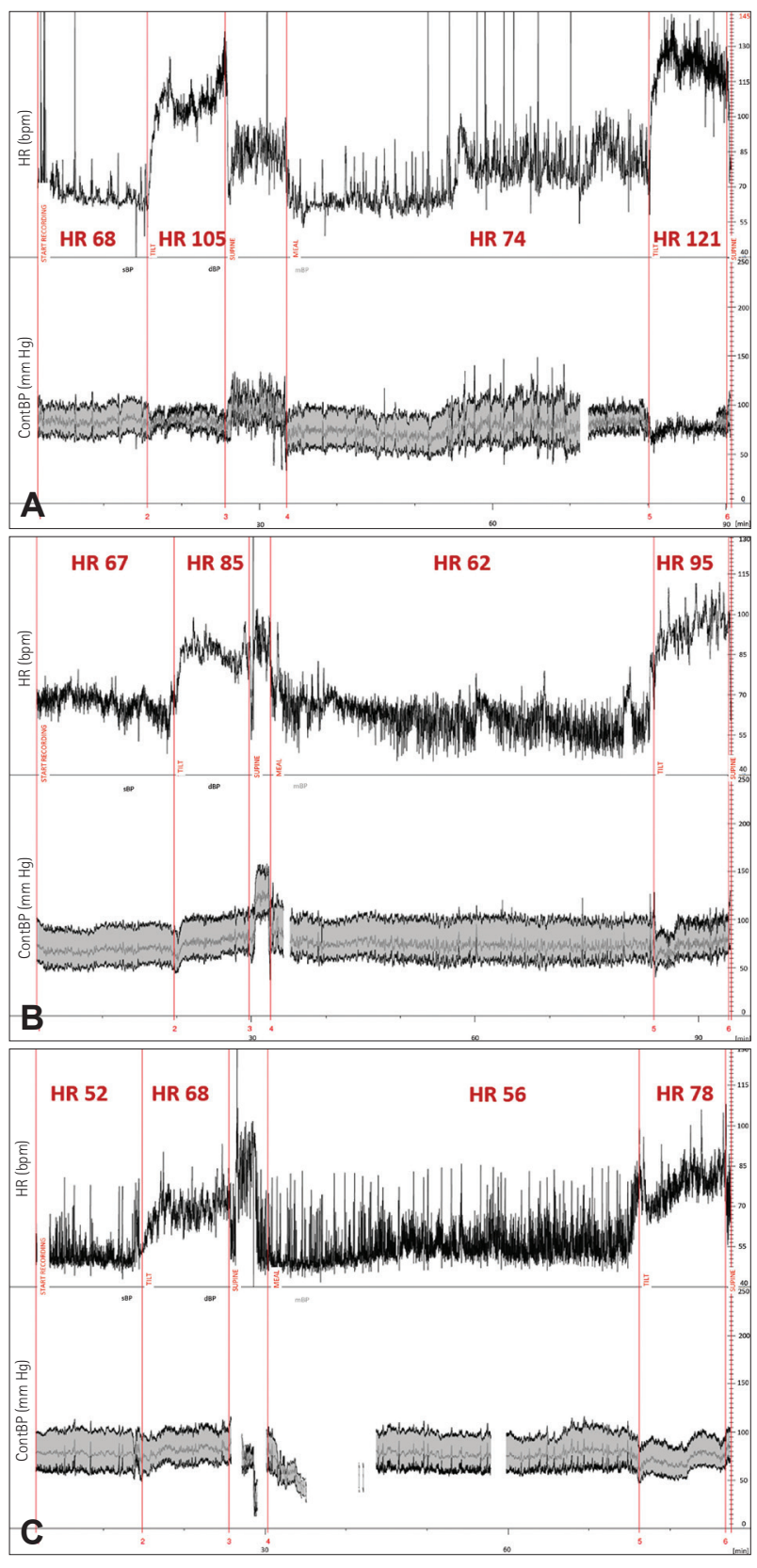

Fig. 2. Example $H R$ and $B P$ tracings from representative patients in groups A (upper part, A), B (middle part, B), and C (lower part, C). ContBP: continuous blood pressure, HR: heart rate.

C were referred to the laboratory due to at least one episode of vasovagal syncope and they had no characteristic POTS symptoms. There were no intergroup differences in age and $\operatorname{sex}(p=0.112$ and $p=0.203$, respectively).

There were no differences in the supine HR and the supine or tilted systolic BP and diastolic BP before or after the meal between the study groups (all $p>0.05$ ). There was a significant difference in the tilted HR before the meal between group A and groups D and C ( $p<0.00001)$. Before the meal, $\Delta$ HR was significantly higher in group A than in all of the other groups, 
Effect of Food Intake on POTS

Table 1. Descriptive data and distribution of HR and BP parameters before and after the ingestion of a meal

\begin{tabular}{|c|c|c|c|c|c|}
\hline & Group A $(n=13)$ & Group B $(n=12)$ & Group C $(n=16)$ & Group D $(n=10)$ & $p$ \\
\hline Age, years & $31.46 \pm 10.53$ & $25.67 \pm 7.89$ & $32.00 \pm 12.06$ & $24.25 \pm 2.25$ & 0.112 \\
\hline Sex, female & 6 & 9 & 13 & 6 & 0.203 \\
\hline \multicolumn{6}{|l|}{ Before meal } \\
\hline \multicolumn{6}{|l|}{$\mathrm{sBP}, \mathrm{mm} \mathrm{Hg}$} \\
\hline Supine & $113.62 \pm 8.47$ & $109.50 \pm 10.42$ & $106.00 \pm 13.35$ & $112.60 \pm 12.17$ & 0.291 \\
\hline Tilted & $109.00 \pm 11.23$ & $109.08 \pm 13.86$ & $110.00 \pm 11.89$ & $109.50 \pm 15.76$ & 0.997 \\
\hline \multicolumn{6}{|l|}{$\mathrm{dBP}, \mathrm{mm} \mathrm{Hg}$} \\
\hline Supine & $72.08 \pm 9.34$ & $63.25 \pm 20.44$ & $66.00 \pm 8.44$ & $68.70 \pm 6.53$ & 0.324 \\
\hline Tilted & $76.77 \pm 11.66$ & $73.00 \pm 9.40$ & $74.19 \pm 9.11$ & $72.90 \pm 9.33$ & 0.751 \\
\hline \multicolumn{6}{|c|}{$\mathrm{HR}$, beats/min } \\
\hline Supine & $74.85 \pm 8.05$ & $75.00 \pm 10.30$ & $68 \pm 10.40$ & $74.50 \pm 16.58$ & 0.311 \\
\hline Tilted & $113.31 \pm 10.01$ & $100.58 \pm 12.99$ & $85.69 \pm 16.33$ & $88.10 \pm 11.87$ & $<0.01^{*}$ \\
\hline \multicolumn{6}{|l|}{ After meal } \\
\hline \multicolumn{6}{|l|}{$\mathrm{sBP}, \mathrm{mm} \mathrm{Hg}$} \\
\hline Supine & $114.23 \pm 8.17$ & $109.08 \pm 8.80$ & $105.19 \pm 11.33$ & $110.10 \pm 13.60$ & 0.165 \\
\hline Tilted & $105.23 \pm 11.88$ & $104.08 \pm 14.10$ & $107.88 \pm 13.45$ & $109.20 \pm 12.28$ & 0.766 \\
\hline \multicolumn{6}{|l|}{$\mathrm{dBP}, \mathrm{mm} \mathrm{Hg}$} \\
\hline Supine & $70.69 \pm 8.47$ & $66.33 \pm 7.33$ & $64.88 \pm 8.69$ & $65.20 \pm 9.71$ & 0.286 \\
\hline Tilted & $72.77 \pm 8.01$ & $68.75 \pm 9.30$ & $72.00 \pm 10.55$ & $69.50 \pm 5.76$ & 0.623 \\
\hline \multicolumn{6}{|c|}{$\mathrm{HR}$, beats/min } \\
\hline Supine & $79.31 \pm 11.54$ & $73.83 \pm 9.86$ & $71.25 \pm 9.50$ & $72.20 \pm 11.87$ & 0.217 \\
\hline Tilted & $125.38 \pm 14.64$ & $107.08 \pm 10.78$ & $92.75 \pm 11.56$ & $95.60 \pm 11.97$ & $<0.01^{+}$ \\
\hline
\end{tabular}

Data are $n$ or mean \pm SD values.

${ }^{*} p<0.01$ for group $A-C$ and group $A-D, p=0.031$ for group $B-C,{ }^{+} p=0.003$ for group $A-B, p<0.01$ for group $A-C$ and group $A-D, p=0.023$ for group $B-C$. $\mathrm{BP}$ : blood pressure, dBP: diastolic blood pressure, HR: heart rate, sBP: systolic blood pressure.

and in group B than in group $\mathrm{D}(p<0.001)$.

Differences in supine HR, tilted HR, and the increase in HR after tilting depending on the ingestion of a meal are presented in Fig. 3.

The results of the ROC analysis performed for $\triangle \mathrm{HR}$ before and after the meal for the POTS and non-POTS groups are presented in Fig. 4. According to the ROC curve of the $\triangle \mathrm{HR}$ before the meal (Fig. 4A), 91\% of the total area was under the curve. To differentiate between subjects with POTS, the optimal cutoff for $\Delta \mathrm{HR}$ was found to be 25 beats $/ \mathrm{min}$, which gave a sensitivity of $92.0 \%$ and a specificity of $80.8 \%$. A cutoff of 30 beats/min for $\Delta$ HR before the meal had a sensitivity of $52.0 \%$ and a specificity of $96.2 \%$.

According to the ROC curve of $\triangle \mathrm{HR}$ after the meal (Fig. 4B), $97 \%$ of the total area was under curve. To differentiate between subjects with POTS, the optimal cutoff for $\Delta \mathrm{HR}$ after the meal was found to be 30 beats $/ \mathrm{min}$, which gave a sensitivity of $96.0 \%$ and a specificity of $96.2 \%$.

\section{DISCUSSION}

The main finding of this study was that the $\Delta \mathrm{HR}$ cutoff for diagnosing POTS depends on the timing of performing the tilt-table test relative to the food intake.

Several previous studies have shown how food ingestion can influence $\triangle \mathrm{HR}$ in patients with POTS, but those studies had different aims. A study performed by Tani et al. ${ }^{11}$ found that there was a significant difference in the tilted HR before and after the meal for both healthy controls and POTS patients ( $77.0 \pm 1.9$ vs. $89.1 \pm 2.7$ beats/min and $120.8 \pm 4.6$ vs. $133.0 \pm 5.4$ beats/min, respectively). Although differences in $\Delta$ HR before and after the meal were not reported for another study, the results showed that the supine HR did not change after the meal in POTS patients; however, during the postprandial orthostatic challenge, POTS patients had a significantly greater $\Delta$ HR compared to healthy subjects. ${ }^{13}$

A particularly interesting observation was that no such influence of food intake on HR was observed in patients with neurally mediated syncope, although a different methodology was used. ${ }^{14}$ Moreover, several studies have suggested that food intake does not significantly change hemodynamic parameters in most healthy people. ${ }^{15,16}$

There are several possible explanations for why such differences are observed in patients with POTS. The splanchnic circulation serves as a large reservoir for blood and is thought to be important for BP regulation. ${ }^{17}$ While normal subjects 


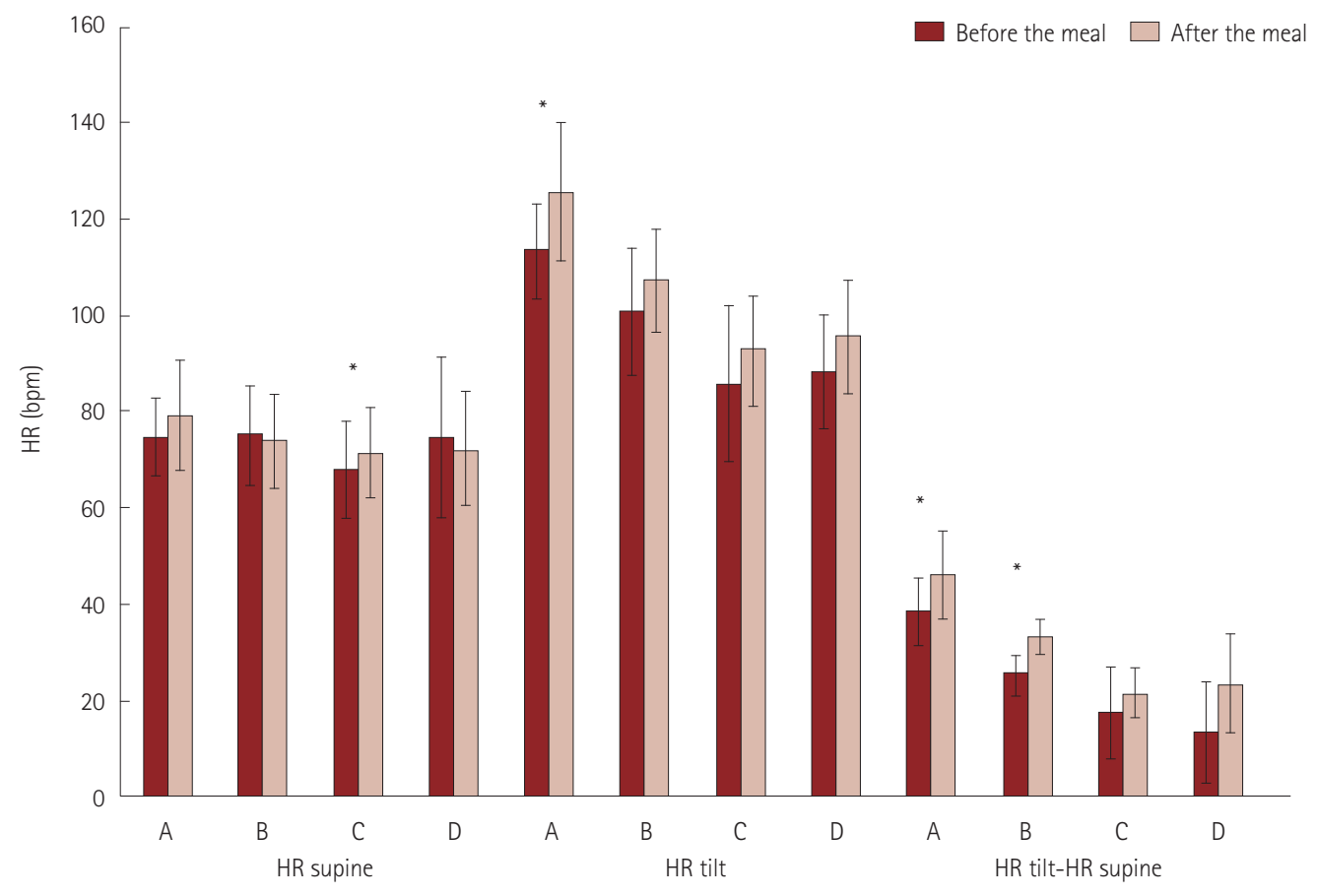

Fig. 3. Differences in supine $H R$, tilted $H R$, and the increase in $H R$ after the tilt depending on the meal. Bar indicate the values before and after the meal, respectively. Note that $\triangle H R$ (difference in HR for standing vs. supine) was significantly higher after the meal only in groups $A$ and $B$ ( $p<0.001$ and $p<0.0001$, respectively). ${ }^{*}$ Indicates significant difference. HR: heart rate.
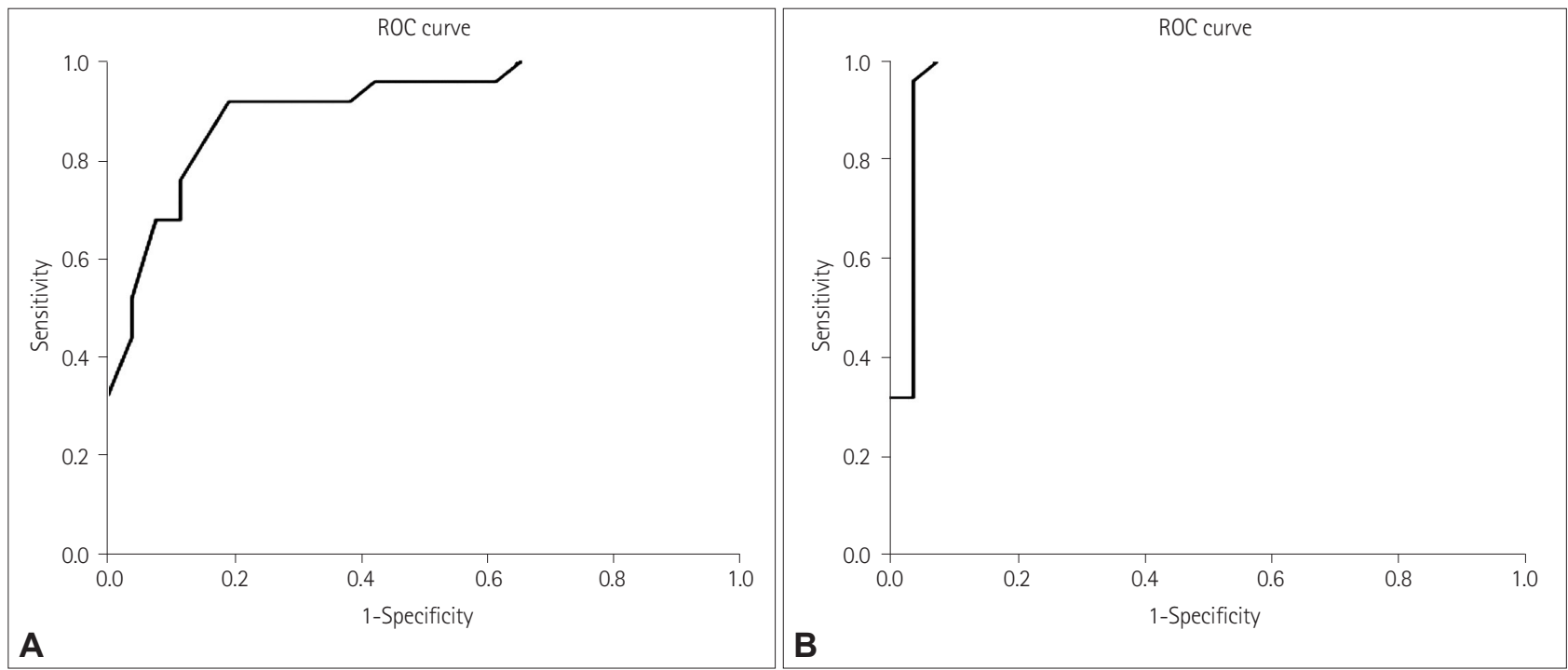

Fig. 4. Results of the ROC analysis performed for $\triangle$ heart rate before (A) and after (B) the meal. ROC: receiver operating characteristics.

compensate for this redistribution of the blood volume that happens after the food ingestion. ${ }^{18}$ patients with chronic generalized autonomic failure do not have recourse to reflex regulation and also develop hypotension and worsening orthostatic hypotension. ${ }^{10,19}$ If POTS is considered to be a mild form of dysautonomia, the increase in HR after food ingestion may be considered a milder consequence of food intake, comparable to the drop in $\mathrm{BP}$ seen in patients with au- tonomic failure. In line with this, postprandial worsening of orthostatic symptoms commonly develops in both conditions. ${ }^{11,20}$

One of the main problems in past research into the effects of food intake on hemodynamic responses in neurological conditions was revealed in a recently performed meta-analysis showing that the nutrients ingested, measurement frequency, duration, and position of the subject are not properly 
defined for the younger neurologic populations that typical suffer from POTS. ${ }^{10}$ It is surprising that almost all such studies use supine HR and BP measurements after meal ingestion, while most of the symptoms and problems of orthostatic intolerance occur in an upright position. Furthermore, the importance of meal ingestion lies in different hemodynamic responses depending on the meal. In patients with neurally mediated syncope, ingesting either carbohydrate or water results in opposite effects on BP during postural maneuvers, whereas this does not occur in control subjects. ${ }^{14}$ Even the osmolality of the fluid may affect the hemodynamic responses in patients with orthostatic intolerance. ${ }^{21}$

One of the limitations of the present study was the possibility of selection bias. Although we enrolled consecutive patients, which patients were referred to the laboratory was dependent on other sources. Furthermore, there is a possibility that some of the patients in group $\mathrm{C}$ recovered by the time they were tested for the second time. Finally, we did not classify patients according to POTS subtypes (e.g., neuropathic or hyperadrenergic).

In conclusion, food intake can significantly alter the results of the tilt-table test and should be taken into account when diagnosing POTS. Performing the tilt-table test after consuming a controlled meal might increase the sensitivity and specificity of the test; however, this hypothesis should be tested in a larger sample of patients and healthy subjects.

\section{Conflicts of Interest}

The authors have no potential conflicts of interest to disclose.

\section{REFERENCES}

1. Garland EM, Celedonio JE, Raj SR. Postural tachycardia syndrome: beyond orthostatic intolerance. Curr Neurol Neurosci Rep 2015;15:60.

2. Raj SR. Postural tachycardia syndrome (POTS). Circulation 2013;127: 2336-2342.

3. Robertson D. The epidemic of orthostatic tachycardia and orthostatic intolerance. Am J Med Sci 1999;317:75-77.

4. Adamec I, Lovrić M, Žaper D, Barušić AK, Bach I, Junaković A, et al. Postural orthostatic tachycardia syndrome associated with multiple sclerosis. Auton Neurosci 2013;173:65-68.

5. Agarwal AK, Garg R, Ritch A, Sarkar P. Postural orthostatic tachycardia syndrome. Postgrad Med J 2007;83:478-480.

6. Freeman R, Wieling W, Axelrod FB, Benditt DG, Benarroch E, Biag- gioni I, et al. Consensus statement on the definition of orthostatic hypotension, neurally mediated syncope and the postural tachycardia syndrome. Clin Auton Res 2011;21:69-72.

7. Trahair LG, Horowitz M, Jones KL. Postprandial hypotension: a systematic review. J Am Med Dir Assoc 2014;15:394-409.

8. Masuda Y, Kawamura A. Role of the autonomic nervous system in postprandial hypotension in elderly persons. J Cardiovasc Pharmacol 2003;42 Suppl 1:S23-S26.

9. Fagius J, Ellerfelt K, Lithell H, Berne C. Increase in muscle nerve sympathetic activity after glucose intake is blunted in the elderly. Clin Auton Res 1996;6:195-203.

10. Pavelić A, Krbot Skorić M, Crnošija L, Habek M. Postprandial hypotension in neurological disorders: systematic review and meta-analysis. Clin Auton Res 2017;27:263-271.

11. Tani H, Singer W, McPhee BR, Opfer-Gehrking TL, Haruma K, Kajiyama G, et al. Splanchnic-mesenteric capacitance bed in the postural tachycardia syndrome (POTS). Auton Neurosci 2000;86:107-113.

12. Crnošija L, Krbot Skorić M, Adamec I, Lovrić M, Junaković A, Mišmaš A, et al. Hemodynamic profile and heart rate variability in hyperadrenergic versus non-hyperadrenergic postural orthostatic tachycardia syndrome. Clin Neurophysiol 2016;127:1639-1644.

13. Seligman WH, Low DA, Asahina M, Mathias CJ. Abnormal gastric myoelectrical activity in postural tachycardia syndrome. Clin Auton Res 2013;23:73-80.

14. Pitt MS, Hainsworth R. Contrasting effects of carbohydrate and water on blood pressure responses to postural maneuvers in patients with posturally related (vasovagal) syncope. Clin Auton Res 2004;14:249254.

15. Imai C, Muratani H, Kimura Y, Kanzato N, Takishita S, Fukiyama K. Effects of meal ingestion and active standing on blood pressure in patients $\geq 60$ years of age. Am J Cardiol 1998;81:1310-1314.

16. Puisieux F, Boumbar Y, Bulckaen H, Bonnin E, Houssin F, Dewailly P. Intraindividual variability in orthostatic blood pressure changes among older adults: the influence of meals. J Am Geriatr Soc 1999;47: 1332-1336.

17. Rowell LB. Regulation of splanchnic blood flow in man. Physiologist 1973;16:127-142.

18. Fujimura J, Camilleri M, Low PA, Novak V, Novak P, Opfer-Gehrking TL. Effect of perturbations and a meal on superior mesenteric artery flow in patients with orthostatic hypotension. J Auton Nerv Syst 1997; 67:15-23.

19. Chaudhuri KR, Thomaides T, Hernandez P, Alam M, Mathias CJ. Noninvasive quantification of superior mesenteric artery blood flow during sympathoneural activation in normal subjects. Clin Auton Res 1991;1:37-42.

20. Mathias CJ, Mallipeddi R, Bleasdale-Barr K. Symptoms associated with orthostatic hypotension in pure autonomic failure and multiple system atrophy. J Neurol 1999;246:893-898.

21. Z'Graggen WJ, Hess CW, Humm AM. Acute fluid ingestion in the treatment of orthostatic intolerance - important implications for daily practice. Eur J Neurol 2010;17:1370-1376. 